\title{
E-ducation and the Languages of Information
}

\author{
Luciano Floridi
}

Published online: 6 August 2013

(C) Springer Science+Business Media Dordrecht 2013

'Civilised', 'cultured', and 'educated': perhaps there were times when these words could rightly be treated as synonymous. Thucydides and Cicero may come to mind. Some characters in Jane Austin, Henry James, or Edith Wharton seem to draw little distinction between the three corresponding concepts. Yet today they hardly overlap at all. 'Civilised' refers to a person's manners and behaviours; 'cultured' qualifies someone who is engaged with arts, letters, and other intellectual pursuits; and 'educated' is usually applied to people who have successfully attended learning or training courses offered by primary, secondary, or tertiary (higher) institutions. One could be any of the three without being either of the remaining two. Globalisation has greatly contributed to this differentiation, even if it has been pushing it in opposite directions, local and global. Montaigne already knew that 'civilised' and 'cultured' had local interpretations. The difference is that today, we feel increasingly less justified in prioritising one 'locality' over the others, be this Rio de Janeiro, New Delhi, Beijing, or Tokyo. We know that it is a matter of civilised manners both to take off one's shoes and to keep them on, it depends on where we are or whom we are visiting. We accept that Alice may be cultured even if she has no clue about Bossa Nova music, Sattriya dance, Sichuanese opera, or Noh plays. And we acknowledge that education is not about any of this. Compulsory schooling, the institutionalisation of teaching and learning, pedagogical universal principles, and the globalisation of the job market began detaching education from upbringing a long time ago. Today, an avionics engineer, a scholar of Mexican literature, a developmental psychologist, or a macro-economist is increasingly evaluated on global, international standards.

Information and communication technologies (ICTs) have further magnified and accelerated both trends. The more they expose us to each other, the more they make us aware that being civilised or cultured is a relative matter. The infosphere (Floridi (2010)) has many nodes but no ultimate centre, so one can only be more or less provincial. However, by making us share needs and expectations on a global scale, ICTs also make us demand similar sets of minimal, educational standards. In the

L. Floridi $(\triangle)$

School of Humanities, University of Hertfordshire, de Havilland Campus, Hatfield, Hertfordshire AL10 9AB, UK e-mail: 1.floridi@herts.ac.uk 
infosphere, being educated is increasingly a de-localised, uniform, and global phenomenon. It is not a relative but a relational matter, in the following sense.

Education is largely about acquisition and transmission of knowledge and of how it may be increased. Broadly construed, the knowledge in question includes not only the critical understanding of facts and formulae, but also the appreciation of values and views, interpretations and perspectives, ways of living and traditions, and abilities and skills. The previous list is incomplete but, in each case, education inevitably relates the educated to something else. The transmitted knowledge may be of a place or a practice, that such and such is or is not the case, of why it may or may not be otherwise, or about how something works or occurs. The solidity of the relation between the educated and the knowledge that is being transmitted is ultimately tested by truth-tracking and truth-generating features. No matter when or where Alice lived, she cannot be said to know Los Angeles, if she has never been there and thinks it is a small Spanish village; she cannot be said to know that the earth is flat or that the kettle is not working because it is Monday; and she cannot be said to know how to drive a car, if she has never driven one in her life.

Of course, history taught in China is quite different from history taught in Japan, and biology courses in the USA are not always comparable to the same courses taught in Britain. So Alice and Bob may easily be educated differently, relatively to the contexts in which they were brought up. The point is that while expectations about being cultured and civilised ought to be relativized, even when they are taken to be absolute, expectations about being educated ought to be absolute, even when they are relativized. This is why it makes sense to compare the success of school children in different countries, but not their level of cultural sophistication, and the quality of universities in the world, but not the degree of the civilised refinement of their students.

Since education is relationally coupled to knowledge, this explains why, when the latter changes, the former must follow suit. Now, hyperhistorical societies (Floridi (2013)) have witnessed the fastest growth of knowledge in the history of humanity. It is a growth that is qualitative and quantitative, both in scope and in pace. Unsurprisingly, the exponential increase of what may be transmitted has caused a major crisis in how we conceive education and organise our pedagogical systems. One widespread and popular reaction has been to try to transform ICTs from being part of the problem to being part of the solution. This is valuable but also distracting. The real educational challenge in hyperhistorical societies is increasingly what to put in the curriculum, not how to teach it (Floridi (2014)). The 'how' is easy, not because it is easily feasible, but because it is more clearly understood. Digital technologies in the classroom are an old phenomenon. A century after Turing's birth, universities are rushing to put their courses online, and the market of e-learning is blooming. There is much to be said in favour of (distance) e-learning, when it is not a form of 'unmanned teaching' or merely cheap outsourcing. As its supporters rightly stress, it has made vast reservoir of educational contents available to millions of people, and it promises to deliver even more to ever more. ICTs may allow a degree of didactic customisation unprecedented in non-elitist contexts: the personalisation of the educational experience for millions of individuals. We know that ICTs are ideal tools to monitor individuals' behaviour. For once, such a power can be put to good use. Yet all this is a matter of delivery policies, methods or techniques, and technologies. If it is taken 
to be a solution of how to educate Generation $Z^{1}$ and the others which will follow, then we are mistaking a painkiller for a cure. The real headache is not the how. Since the late $80 \mathrm{~s}$ we have become enthusiastic about MOOs (text-based online virtual reality systems for multiple users are connected at the same time), literary hypertexts, glove-and-goggle virtual realities, HyperCard, Second Life, and now massive open online courses (MOOCs). The real headache is the what.

There is no clear and fixed answer to the educational 'what' question in hyperhistorical societies, not only because we have never been here before, but also because, like in the past, the answer still depends on the answer to another question, the 'what for' of e-ducation. Nevertheless, a few considerations may set some boundaries within which we can search for a solution.

Suppose Alice is playing a computer game. There are things that she knows, e.g., there is a monster hiding. This is her knowledge. There are things that she knows that she does not know, e.g., where the monster is hiding, and that is why she is searching for it. This is her lack of knowledge or simply insipience. There are things that she is not quite sure she knows, e.g., whether her weapons are sufficiently powerful to kill the monster, and that is why she is trying to acquire some more. This is her uncertainty. And there are things that she does not even know that she does not know: there is a magic sword that can kill the monster. This is her ignorance. We can translate the example into informational terms, thus:

(a) information Alice has (there is a monster)

(b) information that Alice is aware she is missing (where is the monster hiding?)

(c) information about which Alice is uncertain (are my weapons sufficient to kill the monster?)

(d) information that Alice is not aware she is missing (if only she knew that she is missing the fact that there is a magic weapon!)

Education has always had the goal of increasing (a) and decreasing (b), (c), and (d).

Regarding (a), in a world awash with easily accessible information, cheap ICTs, and a plentiful intellectual workforce, increasing knowledge has become easy, hence the success of MOOCs based on interactive participation and open access through the web. The educational problem with (a) is that new information always requires some old, background information to become meaningful and useful. So we need to

\footnotetext{
${ }^{1}$ Sociologists speak of Generation X-people with birth dates from the early 1960 s to the early 1980 s - and of Generation Y, or Millennial Generation, which includes people with birth dates from the early 1980 s to 2000 or so. I refer to people born after the long nineties-long because they lasted until September 112001 - as Generation Z, not just because of the previous two alphabetical generations X and $\mathrm{Y}$, but also because of the Zettabyte data available to them for the first time in the history of humanity. To anyone belonging to Generation Z, the world has always been wireless everywhere; $9 / 11$ is a chapter in their sixth grade history book. For them, there has never been a world without 'google', 'twit' and 'wiki' as verbs, they have no recollection of a world without Facebook being a social media, not a book, and of books not available online (Amazon was incorporated in 1994). They use 'Wikipedia' (founded in 2001) as synonymous with encyclopaedia. To Generation $\mathrm{Z}$, the peculiar clicking and whooshing sounds made by conventional modems while handshaking, also known as the whale song, is as archaeologically alien as the sounds made by a telegraph's Morse signals is to us. They cannot conceive life outside the infosphere because, to put it dramatically, the infosphere is progressively absorbing any other ontological space and they were born onlife.
} 
understand how much and what kind of background information - things you need to know, independently of whether you may check them on Wikipedia-Alice needs in order to be educated today.

Regarding (b), education should teach us the limits of our knowledge, what kind of information we do not have but might want to acquire, and hence a good taste for the right sort of questions we should ask. We are all insipient, it is how we handle our degree of insipience that makes a difference. So, the educational problem with (b) becomes what kind of unknowns Alice should be taught today.

Regarding (c), education should teach us to be careful about what we think we know, and hence the art of doubting and being critical even of the seemingly certain. We are all fallible, it is how we handle our degree of fallibility that makes a difference. So, the educational problem with (c) becomes what kind of uncertainties Alice should be taught today.

As for (d), it is an internal problem. This is why only we can describe it for Alice. If Alice knew what she does not know that she is missing, she would be insipient or uncertain about it, not ignorant, after all. Now, imagine that we could talk to Alice: at a stroke, we could tell her that she is missing some information about the existence of a magic sword, so that particular instance of her ignorance would be erased. This is what a more globalised education across geographical borders and academic boundaries can do. It cannot erase humanity's ignorance, but it can place each human being on one side of the same divide, even if, by definition, we, as humanity, do not know where that divide is. Let me explain using the same example. Suppose Bob knows that he does not know where the magic sword is, but he is not even aware of missing the information that there is a monster nearby. If Alice and Bob share their insipience, then they can decrease their ignorance: together they will know that they do not know where the monster is and where the magic sword is. It may sound funny, but this is a great improvement. Internal ignorance is decreased, even if external ignorance (what Alice and Bob as a group are unaware that they are missing, imagine both of them being ignorant about the existence of a friendly wizard) may not be.

In what I have said so far, the tension between facts and skills remains. Is it more important to teach Alice that the monster has seven heads, all of which need to be cut in order to kill it, or to teach Alice how to cut them? You immediately see the misleading nature of the facts vs. skills dichotomy. She needs to have both kinds of knowledge, or she will not win the game. However, today, because so much information is just a click away, there seems a tendency to privilege know-how over know-that. This is silly, especially if one recalls the importance of background information stressed above. It is also misleading, if by privileging know-how we promote a culture of users and consumers, instead of a culture of designers and producers as well. A hyperhistorical society is a neo-manufacturing society in which information is both the raw material we produce and manipulate and the finished good we consume. In such a society, when it comes to skills, we really need to place more emphasis than ever before on the so-called maker's knowledge, the knowledge that is enjoyed by those who know how to design and produce the artefacts, that is, those who know how to create, design, and transform information. This is easier said than done, since 
our Western culture is based on a Greek divide between episteme (knowledge that), which is highly valued and respected, and techne (knowledge how), which is seen as secondary. Not only it is a false dichotomy, as we just saw with Alice and the monster, it is also one that focuses too much on the wrong side of the coin. Using our previous example, the game of knowledge includes players, watchers, and designers. A fact-based education and a skillbased education are strategies for players. They both address informational organisms (inforgs) like us only as users, not also as producers of information. The risk is to develop a 'luxury box' reaction, with watchers enjoying the knowledge game without actually playing it. It used to be called an ivory tower. Meanwhile, an important part of the real business of education takes place at the game-designer level.

We need to teach Alice-the-user how to play the game successfully, Alice-theintellectual how to observe and study the game critically, and Alice-the-designer how to devise the whole game properly. So the question becomes: what sort of abilities should we privilege and teach to tomorrow's consumers, curators, producers, and designers of information? The answer seems to me quite obvious: the very languages through which information is created, manipulated, accessed, and consumed. By this, I do not mean only one's own natural language, the full mastery of which is the first, basic, necessary step for any other form of education. I also mean English (or whatever language will one day be the international medium of communication), mathematics, programming, music, graphics, and all those natural and artificial languages in which inforgs need to be proficient at a very early stage of their development, in order to be able to understand and use critically the accessible information, create and design new information, and share it with others. Languages are best learnt when we are young. And language proficiency is not a matter of memorised facts or practised skills, but of finely tuned abilities.

ICTs are great in making information available; they are less successful in making it accessible, and even less so in making it usable. Try reading a scientific entry (pick your science) on Wikipedia and the likelihood is that much of it will be impenetrable, if you do not speak the right language. More availability and better accessibility are issues on the side of the providers. But information production and design at the very beginning of the availability process, and usability and comprehension of the accessible information at the end, are issues that involve the education of the inforgs involved. And this means that Alice needs to learn to speak the language of information. ICTs are going to increase the demand for e-ducators.

\section{References}

Floridi, L. (2010). Information - a very short introduction. Oxford: Oxford University Press.

Floridi, L. (Ed.). (2014). The onlife manifesto. New York: Springer. in press.

Floridi, L. (2013). The fourth revolution - on the impact of information and communication technologies on our lives. Oxford: Oxford University Press. in press. 